\title{
Impact of Polypharmacy on Health-Related Quality of Life in Dialysis Patients
}

\author{
Julia M.T. Colombijn ${ }^{a} \quad$ Anna A. Bonenkamp ${ }^{a} \quad$ Anita van Eck van der Sluijs ${ }^{b}$ \\ Joost A. Bijlsma ${ }^{a, c}$ Arnold H. Boonstra ${ }^{d}$ Akin Özyilmaz ${ }^{\mathrm{e}}{ }^{\mathrm{f}}$ Alferso C. Abrahams ${ }^{\mathrm{b}}$ \\ Brigit C. van Jaarsveld ${ }^{a, g}$ on behalf of the DOMESTICO study group \\ aDepartment of Nephrology, Amsterdam UMC, Research Institute Amsterdam Cardiovascular Sciences, Vrije Universiteit \\ Amsterdam, Amsterdam, The Netherlands; ${ }^{b}$ Department of Nephrology and Hypertension, University Medical Centre

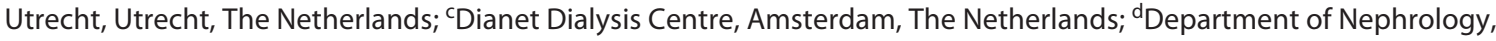 \\ Flevoziekenhuis, Almere, The Netherlands; ${ }^{e}$ Dialysis Centre Groningen, Groningen, The Netherlands; ${ }^{f}$ Division of \\ Nephrology, Department of Internal Medicine, University Medical Center Groningen, Groningen, The Netherlands; \\ gDiapriva Dialysis Centre, Amsterdam, The Netherlands
}

\section{Keywords}

Polypharmacy · Health-related quality of life · Quality of life · Dialysis

\begin{abstract}
Introduction: Dialysis patients are often prescribed a large number of medications to improve metabolic control and manage coexisting comorbidities. However, some studies suggest that a large number of medications could also detrimentally affect patients' health-related quality of life (HRQoL). Therefore, this study aims to provide insight in the association between the number of types of medications and HRQoL in dialysis patients. Methods: A multicentre cohort study was conducted among dialysis patients from Dutch dialysis centres 3 months after initiation of dialysis as part of the ongoing prospective DOMESTICO study. The number of types of medications, defined as the number of concomitantly prescribed types of drugs, was obtained from electronic patient records. Primary outcome was HRQoL measured with the Physical Component Summary (PCS) score and Mental Component Summary (MCS) score (range 0-100) of the Short Form 12. Secondary outcomes were number of symptoms (range 0-30) measured with the Dialy-
\end{abstract}

sis Symptoms Index and self-rated health (range 0-100) measured with the EuroQol-5D-5L. Data were analysed using linear regression and adjusted for possible confounders, including comorbidity. Analyses for MCS and number of symptoms were performed after categorizing patients in tertiles according to their number of medications because assumptions of linearity were violated for these outcomes. $\boldsymbol{R e}$ sults: A total of 162 patients were included. Mean age of patients was $58 \pm 17$ years, $35 \%$ were female, and $80 \%$ underwent haemodialysis. The mean number of medications was $12.2 \pm 4.5$. Mean PCS and MCS were $36.6 \pm 10.2$ and $46.8 \pm 10.0$, respectively. The mean number of symptoms was $12.3 \pm 6.9$ and the mean self-rated health $60.1 \pm 20.6$. In adjusted analyses, PCS was 0.6 point lower for each additional medication ( $95 \%$ confidence interval [ $95 \% \mathrm{Cl}]:-0.9$ to $-0.2 ; p=0.002)$. MCS was 4.9 point lower $(95 \% \mathrm{Cl}:-8.8$ to $-1.0 ; p=0.01)$ and 1.0 point lower $(95 \% \mathrm{Cl}:-5.1-3.1 ; p=0.63)$ for the highest and middle tertiles of medications, respectively, than for the lowest tertile. Patients in the highest tertile of medications reported 4.1 more symptoms than in the lowest tertile (95\% Cl: $1.5-6.6 ; p=0.002)$, but no significant difference in the number of symptoms was observed between the middle and lowest tertiles. Self-rated health was 1.5 point lower for each medication $(95 \% \mathrm{Cl}:-2.2$ to $-0.7 ; p$
(C) 2021 The Author(s)

Published by S. Karger AG, Basel

This is an Open Access article licensed under the Creative Commons Attribution-NonCommercial-4.0 International License (CC BY-NC) (http://www.karger.com/Services/OpenAccessLicense), applicable to the online version of the article only. Usage and distribution for commercial purposes requires written permission.
Correspondence to:

Brigit C. van Jaarsveld, b.jaarsveld@ amsterdamumc.nl 
< 0.001). Discussion/Conclusion: After adjustment for comorbidity and other confounders, a higher number of medications were associated with a lower PCS, MCS, and self-rated health in dialysis patients and with more symptoms.

(c) 2021 The Author(s)

Published by S. Karger AG, Basel

\section{Introduction}

Patients with end-stage kidney disease who undergo dialysis report a significantly lower health-related quality of life (HRQoL) than the general population [1]. Moreover, their HRQoL is worse than the HRQoL of kidney transplant recipients and patients with other chronic diseases such as diabetes, congestive heart failure, malignancies, and chronic lung disease $[1,2]$.

Several factors could contribute to this diminished HRQoL of dialysis patients. First, dialysis patients commonly suffer from numerous somatic and mental healthrelated symptoms attributable to kidney failure or kidney failure-related comorbidity such as dyspnoea, pruritus, fatigue, sleeping disorders, anxiety, and depression $[3,4]$. Second, stringent dietary and other lifestyle restrictions to prevent accumulation of fluid and electrolytes and to improve metabolic control can also diminish HRQoL [5].

In addition, dialysis treatment itself can detrimentally affect patients' HRQoL. First, dialysis severely restricts patients' freedom since multiple dialysis sessions per week usually are required to sufficiently compensate a loss of kidney function. Second, dialysis-related complications such as infections or problems with dialysis access can arise which require hospitalization or additional treatment $[6,7]$. Third, patients undergoing haemodialysis frequently suffer from intradialytic and post-dialytic symptoms such as fatigue, hypotension, and muscle cramps [8].

Studies in various patient populations, including kidney transplant recipients and geriatric patients, suggest that polypharmacy can also negatively affect HRQoL [9, 10]. This may be due to complexity of medication regimens and medication-related problems such as adverse events [11-13]. Exposure to polypharmacy seems unavoidable for dialysis patients since they invariably require multiple medications to treat the course of kidney failure and coexisting comorbidity and to correct ensuing metabolic disturbances $[14,15]$.

Despite this consequent exposure to polypharmacy among dialysis patients, only a handful of studies to date have evaluated the association between polypharmacy and HRQoL in this patient population, of which only one study particularly focussed on polypharmacy and HRQoL [16-18]. Therefore, the primary aim of this study is to explore the association between the number of types of medication and HRQoL in dialysis patients.

\section{Materials and Methods}

\section{Study Design}

We conducted a cross-sectional multicentre cohort study among dialysis patients as part of the ongoing prospective Dutch nOcturnal and hoME dialysis Study To Improve Clinical Outcomes (DOMESTICO) [19]. DOMESTICO is a prospective international cohort study in the Netherlands and Belgium that longitudinally compares HRQoL and clinical outcomes of home dialysis and in-centre haemodialysis patients. For the present study, we used medication and HRQoL data at 3 months after the start of dialysis. Patients are eligible to be included in DOMESTICO if they are at least 18 years old and diagnosed with end-stage kidney disease for which they have to (re)start with haemodialysis or peritoneal dialysis. Exclusion criteria are a life expectancy shorter than 3 months and an expected kidney transplant within 3 months [19]. Patients have been included in DOMESTICO from December 2017 onwards.

\section{Study Population}

The study population consisted of a subset of DOMESTICO patients at 3 months after dialysis initiation from 7 Dutch dialysis centres: 4 university hospitals, 2 community hospitals, and 1 standalone dialysis centre. Participating centres were selected based on the availability of complete data on patients' prescribed medication. Patients were screened for inclusion until July 2020. Additional exclusion criteria for this study were no available medication list at 3 months or a missing Short Form 12 (SF-12), which is the primary outcome. Patients were secondarily excluded if they had filled out less than $75 \%$ of the SF- 12 .

\section{Medication Measurements}

Medication data were retrieved from patients' electronic patient files. The number of medications was defined as the number of concomitantly prescribed types of drugs. All medication that was prescribed for at least 14 consecutive days was listed, including prescribed-as-needed and non-systemic medication. Drugs from the same pharmacological subgroup, for example, insulin glargine and insulin aspart, were counted as separate medications. Singlepill combinations were counted as the number of drugs included (for example, lisinopril/hydrochlorothiazide was considered as 2 types of medication) for 2 reasons: first, single-pill combinations consist of multiple medications that each have their own pharmacodynamics that can affect HRQoL. Counting these medications as separate medications fits our study objective best as we investigate the association between the number of types of prescribed medications, and HRQoL rather than daily pill burden. Furthermore, in The Netherlands, it depends on the prescribing physician and pharmacy if medication is dispensed to patients as separate medications or in a combination medication. Including single-pill combinations as the number of included drugs ensures that all medication is weighed equally irrespective of the prescribing physician's and pharmacist's decision to prescribe medication as a sin- 
gle-pill combination or as separate medications. Over-the-counter drugs and herbal medicines were excluded to avoid misclassification bias [20]. Medications administered during dialysis were also excluded. Polypharmacy was defined as the concurrent prescription of 5 or more types of medication [21].

\section{HRQoL Measurement}

HRQoL was assessed using 3 self-reported questionnaires, which have been validated for dialysis patients [22, 23]: the SF-12, the Dialysis Symptom Index (DSI), and the EuroQoL-5D-5L (EQ5D-5L). The SF-12 and EQ-5D-5L are generic quality of life questionnaires, and the DSI is a dialysis-specific questionnaire. Patients filled out questionnaires online or on paper, depending on their preference. If patients' 3-month questionnaire was missing, their 6-month questionnaire was used as a substitute.

The primary outcome was HRQoL measured with the Physical Component Summary (PCS) and Mental Component Summary (MCS) scores of the SF-12 [24]. The SF-12 is a condensed version of the Short Form 36 comprising 12 of its items from which PCS and MCS scores are calculated [25]. Scores were standardized to US general population scores with a mean of 50 and a standard deviation of 10 . Scores range from 0 to 100 with higher scores indicating better HRQoL. A difference of 3 points in SF-12 summary scores was considered clinically important [26, 27].

Secondary HRQoL outcomes were the number of symptoms and total symptom burden measured with the DSI and EQ-5D-5L utility score and self-rated health measured with the EQ-5D-5L. The DSI assesses the prevalence and severity of 30 dialysis-related symptoms [28]. Patients indicate per symptom whether they experienced it during the past week and, if so, its severity on a 5-point Likert scale ranging from 1 ("not at all bothersome") to 5 ("very bothersome"). The number of symptoms (range 0-30) is the number of times a patient answered "yes" to an item. The total symptom burden (range $0-150$ ) is the sum of the Likert scale scores. Higher scores indicate a higher symptom burden.

The EQ-5D-5L rates patients' HRQoL on 5 domains [29]. Each domain is scored on a 5-point Likert scale from which utility scores were calculated using the Dutch value set (range 0-1) [30]. In addition, the EQ-5D-5L measures patients' self-rated health on a visual analogue scale (range 0-100). Higher scores indicate better HRQoL and self-rated health.

\section{Collection of Covariates}

Patient demographics, medical history, and clinical parameters were collected from electronic patient files and questionnaires. Cause of kidney failure was categorized based on the classification of the European Renal Association - European Dialysis and Transplant Association (ERA-EDTA) [31]. Comorbidity scores were calculated using the Charlson Comorbidity Index (CCI), which includes the presence and severity of a wide range of conditions [32].

\section{Statistical Analyses}

Categorical variables are presented as percentages and continuous variables as mean \pm standard deviation (SD) or median and interquartile range, depending on whether they are normally distributed. The association between the number of medications and HRQoL was analysed using linear regression. Assumptions of linearity were violated for MCS score, number of symptoms, total symptom burden, and EQ-5D-5L utility scores. These outcomes

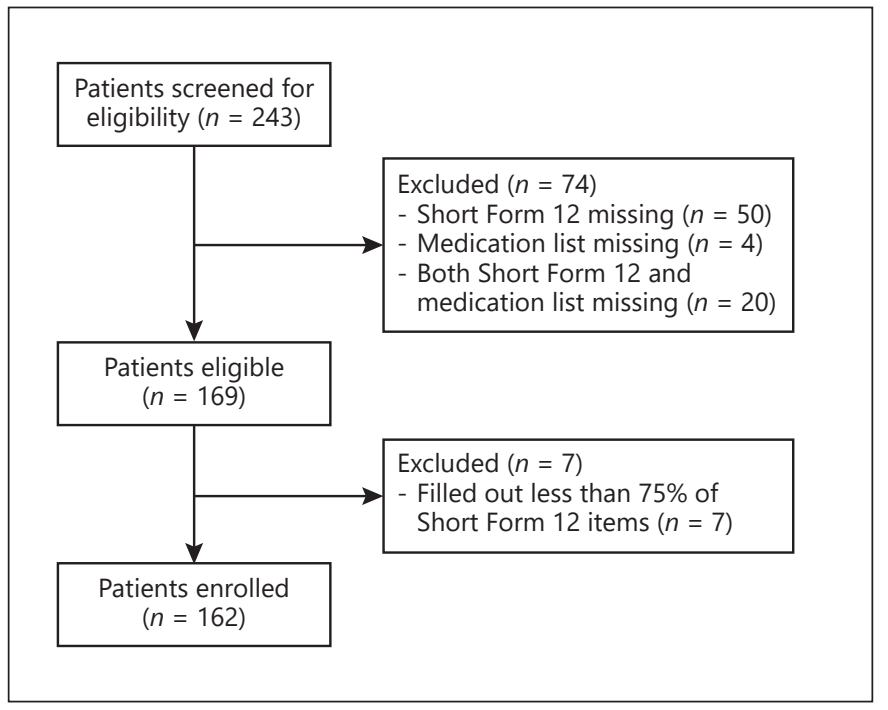

Fig. 1. Flowchart of patient selection.

were therefore analysed after categorizing patients in tertiles of medications as using existing classifications of polypharmacy would have resulted in significantly unequal groups. Patient characteristics across different tertiles were compared using analysis of variance, Kruskal-Wallis test, or $\chi^{2}$ test.

Adjustment for confounding was based on 2 models: model 1 was adjusted for age and sex and model 2 was further adjusted for comorbidity according to CCI, dialysis modality, acute start of dialysis, and hospitalization in the past 3 months. Outcomes were considered statistically significant for $p \leq 0.05$.

Missing data were imputed using multiple imputation techniques. Twenty imputed datasets were created through predictive mean matching. Missing data in questionnaires were imputed at item level instead of score level from which total scores were calculated [33]. Multiple imputation was not conducted for questionnaires which missed more than $25 \%$ of questionnaire items.

\section{Sensitivity Analyses}

To further explore the robustness of our results, 2 sensitivity analyses were performed for both crude and adjusted analyses. The impact of multiple imputation was investigated by conducting an analysis of patients with complete questionnaires (complete case analysis).The impact of using 6-month questionnaires was investigated by including only patients with a 3 -month questionnaire. All data were analysed in SPSS (version 26.0).

\section{Results}

\section{Patient Selection}

In total, 243 patients were screened for eligibility, of whom 162 were included (Fig. 1). Main reasons for exclusion were missing SF-12 $(n=50)$ or missing SF-12 and medication list $(n=20)$ (Fig. 1$)$. 
Table 1. Patient characteristics for all patients and per medication tertile

\begin{tabular}{|c|c|c|c|c|}
\hline & $\begin{array}{l}\text { All patients } \\
(n=162)\end{array}$ & $\begin{array}{l}2-10 \text { medications } \\
(n=59)\end{array}$ & $\begin{array}{l}11-13 \text { medications } \\
(n=42)\end{array}$ & $\begin{array}{l}14-27 \text { medications } \\
(n=61)\end{array}$ \\
\hline \multicolumn{5}{|l|}{ Demographic data } \\
\hline Sex (female) & $56(35)$ & $22(37)$ & $12(29)$ & $22(36)$ \\
\hline \multicolumn{5}{|l|}{ Medical history } \\
\hline \multicolumn{5}{|l|}{ Cause of kidney failure ${ }^{a}$} \\
\hline Renovascular nephropathy & $44(27)$ & $15(25)$ & $15(36)$ & $14(23)$ \\
\hline Diabetic nephropathy & $26(16)$ & $3(5)$ & $8(19)$ & $15(25)$ \\
\hline Other/unknown & $39(24)$ & $19(32)$ & $7(17)$ & $13(21)$ \\
\hline Previous dialysis & $20(12)$ & $4(7)$ & $7(17)$ & $9(15)$ \\
\hline Dialysis vintage ${ }^{\mathrm{b}}$ (months) & $32.5(11.8-64.3)$ & $32.0(8.3-64.0)$ & $21.0(7.0-67.0)$ & $51.0(18.0-77.5)$ \\
\hline Previous kidney transplant & $37(23)$ & $10(17)$ & $11(26)$ & $16(26)$ \\
\hline Haemodialysis & $130(80)$ & $49(83)$ & $34(81)$ & $47(77)$ \\
\hline Peritoneal dialysis & $32(20)$ & $10(17)$ & 8 (19) & $14(23)$ \\
\hline Acute start of dialysis & $23(14)$ & $14(24)$ & $4(10)$ & $5(8)$ \\
\hline \multicolumn{5}{|l|}{ Clinical parameters } \\
\hline $\mathrm{BMI}, \mathrm{kg} / \mathrm{m}^{2}$ & $26.6 \pm 6.4$ & $26.5 \pm 6.6$ & $26.2 \pm 5.5$ & $26.8 \pm 6.9$ \\
\hline Residual diuresis $^{c}$ & $141(97)$ & $51(100)$ & $38(100)$ & $52(93)$ \\
\hline Haemoglobin, mmol/L & $6.8 \pm 1.0$ & $6.9 \pm 1.2$ & $6.9 \pm 1.0$ & $6.8 \pm 0.9$ \\
\hline Phosphate, $\mathrm{mmol} / \mathrm{L}$ & $1.73 \pm 0.53$ & $1.79 \pm 0.55$ & $1.68 \pm 0.50$ & $1.72 \pm 0.54$ \\
\hline Calcium, mmol/L & $2.26 \pm 0.21$ & $2.27 \pm 0.18$ & $2.31 \pm 0.15$ & $2.22 \pm 0.25$ \\
\hline Albumin, g/L & $37.5 \pm 6.4$ & $38.5 \pm 5.0$ & $37.2 \pm 5.5$ & $36.8 \pm 8.0$ \\
\hline \multicolumn{5}{|l|}{ Medication use } \\
\hline Medications (mean), $n$ & $12.2 \pm 4.5$ & $7.6 \pm 2.0$ & $12.0 \pm 0.9$ & $16.7 \pm 2.9$ \\
\hline EQ-5D-5L utility score & $0.71 \pm 0.24$ & $0.80 \pm 0.18$ & $0.75 \pm 0.23$ & $0.61 \pm 0.27$ \\
\hline Self-rated health & $60.1 \pm 20.6$ & $66.7 \pm 18.4$ & $62.2 \pm 20.5$ & $52.1 \pm 20.2$ \\
\hline
\end{tabular}

Continuous variables are presented as mean \pm standard deviation or median [interquartile range] and categorical variables as $n$ (\%). The sum of percentages may not equal 100 due to rounding off to the nearest integer. Reported values are from the non-imputed dataset. Missing data: BMI was missing for 22 participants (14\%), residual diuresis for 17 (10\%), albumin for 10 (6\%), hospitalization in the past 3 months for $8(5 \%)$, haemoglobin and phosphorus for $7(4 \%)$, and calcium for $6(4 \%)$. Other variables contained no missing data. SF-12 was complete for 139 patients (86\%), DSI for 121 (75\%), and EQ-5D-5L for 150 (93\%). BMI, body mass index; SF-12, Short Form

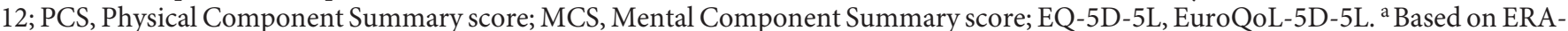
EDTA codes. ${ }^{\mathrm{b}}$ Only for patients who have previously undergone dialysis. ${ }^{\mathrm{c}}$ Defined as the urine production $>100 \mathrm{~mL} /$ day. ${ }^{\mathrm{d}}$ Defined as the concurrent use of 5 or more medications.

\section{Patient Characteristics}

Patient characteristics are listed in Table 1. Mean age was $58 \pm 17$ years, 56 patients (35\%) were female, 130 (80\%) underwent haemodialysis, and $32(20 \%)$ peritoneal dialysis. The mean number of medications was $12.2 \pm$
4.5 (range 2-27), and 158 patients (98\%) met the criteria for polypharmacy. Categorizing patients in tertiles resulted in tertiles in which the number of medications ranged from 2 to 10 (mean 7.6), 11 to 13 (mean 12.0), and 14 to 27 (mean 16.7). Patients with $11-13$ and 14-27 medica- 
Table 2. Association between the number of types of medication and HRQoL

\begin{tabular}{|c|c|c|c|c|c|c|}
\hline & RC (95\% CI) & $p$ value & $\mathrm{RC}(95 \% \mathrm{CI})$ & $p$ value & $\mathrm{RC}(95 \% \mathrm{CI})$ & $p$ value \\
\hline \multicolumn{7}{|l|}{ Short Form 12} \\
\hline PCS & $-0.7(-1.0$ to -0.4$)$ & $<0.001$ & $-0.7(-1.0$ to -0.3$)$ & $<0.001$ & $-0.6(-0.9$ to -0.2$)$ & 0.002 \\
\hline \multicolumn{7}{|l|}{$\mathrm{MCS}^{\mathrm{c}}$} \\
\hline $2-10$ types of medications & REF & & REF & & REF & \\
\hline \multicolumn{7}{|l|}{ Dialysis symptom index } \\
\hline \multicolumn{7}{|l|}{ Symptoms, $n^{\mathrm{c}}$} \\
\hline 2-10 types of medications & REF & & REF & & REF & \\
\hline $11-13$ types of medications & $-1.3(-3.8-1.3)$ & 0.32 & $-0.9(-3.5-1.7)$ & 0.49 & $-1.2(-3.9-1.5)$ & 0.37 \\
\hline 14-27 types of medications & $4.4(2.2-6.7)$ & $<0.001$ & $4.6(2.3-6.8)$ & $<0.001$ & $4.1(1.5-6.6)$ & 0.002 \\
\hline \multicolumn{7}{|l|}{ Total symptom burden ${ }^{c}$} \\
\hline \multicolumn{7}{|l|}{ EQ-5D-5L utility score ${ }^{\mathrm{c}}$} \\
\hline 2-10 types of medications & REF & & REF & & REF & \\
\hline $11-13$ types of medications & $-0.05(-0.14-0.05)$ & 0.32 & $-0.06(-0.15-0.04)$ & 0.24 & $-0.05(-0.15-0.05)$ & 0.31 \\
\hline $14-27$ types of medications & $-0.20(-0.28$ to -0.12$)$ & $<0.001$ & $-0.20(-0.29$ to -0.12$)$ & $<0.001$ & $-0.19(-0.28$ to -0.09$)$ & $<0.001$ \\
\hline Self-rated health & $-1.6(-2.3$ to -1.0$)$ & $<0.001$ & $-1.7(-2.4$ to -1.0$)$ & $<0.001$ & $-1.5(-2.2$ to -0.7$)$ & $<0.001$ \\
\hline
\end{tabular}

Two patients were omitted from the DSI analyses and 4 from the EQ-5D-5L analyses because they missed more than $25 \%$ of items. RC, regression coefficient; REF, reference category; PCS, Physical Component Summary Score; MCS, Mental Component Summary score; EQ-5D-5L, EuroQoL-5D-5L. ${ }^{a}$ Model 1: adjusted for age and sex. ${ }^{\mathrm{b}}$ Model 2: further adjusted for Charlson Comorbidity Index, dialysis modality, acute start of dialysis, and hospitalization in the past 3 months. ${ }^{c}$ Analysed with a number of medications categorized in tertiles due to violation assumptions of linearity.

tions had a higher CCI and a higher prevalence of diabetic nephropathy than patients with $2-10$ medications. The prevalence of cystic kidney disease and acute start of dialysis was higher among patients with 2-10 medications than patients with $11-13$ and 14-27 medications.

\section{HRQoL Scores}

Patients' HRQoL scores are listed in Table 2. The SF12 was filled out completely for 139 patients (86\%), the DSI for 121 (75\%), and the EQ-5D-5L for 151 (93\%). Sixmonth instead of 3-month questionnaires were used for 22 patients. Mean PCS and MCS scores were $36.6 \pm 10.2$ and $46.8 \pm 10.0$, respectively. Mean number of symptoms was $12.3 \pm 6.9$ and mean symptom burden $33.2 \pm 21.9$. Mean EQ-5D-5L utility score was $0.71 \pm 0.24$ and mean self-rated health $60.1 \pm 20.6$. Mean PCS and MCS scores, EQ-5D-5L utility score, and self-rated health were lower for subsequent tertiles. Mean number of symptoms and total symptom burden were highest for patients with 1427 medications and lowest for patients with 11-13 medications.

Polypharmacy and Quality of Life Dialysis Patients

\section{Association between the Number of Types of Medication and HRQoL}

The results of the analyses are illustrated in Figure 2 and summarized in Table 2. Results of the crude and adjusted analyses were comparable. In the adjusted analyses, PCS score was 0.6 point (95\% confidence interval [95\% CI]: -0.9 to -0.2 ) lower for each additional medication. MCS score was 4.9 (95\% CI: -8.8 to -1.0$)$ and 1.0 point (95\% CI: -5.1 to -3.1 ) lower for patients with $14-27$ and $11-13$ medications, respectively, than patients with 2-10 medications. In addition, patients with 14-27 medications reported 4.1 more symptoms (95\% CI: $1.5-6.6$ ) and a 14.8 point higher total symptom burden (95\% CI: 6.9-22.7) than patients with 2-10 medications. Patients with 14-27 medications also reported 0.19 (95\% CI: -0.28 to -0.09 ) point lower EQ-5D-5L utility scores than patients with 2-10 medications. Self-rated health was 1.5 point lower (95\% CI: -2.2 to -0.7 ) for each additional medication. No statistically significant difference was observed in the number of symptoms, total symptom burden, and EQ-5D-5L utility score between patients with 11-13 and 2-10 medications. 
Fig. 2. PCS scores (a), MCS scores (b), number of symptoms (c), total symptom burden (d), EQ-5D-5L utility score (e), and self-rated health (f) per number of medications for adjusted analyses. PCS, Physical Component Summary; MCS, Mental Component Summary; EQ-5D-5L, EuroQoL-5D-5L.
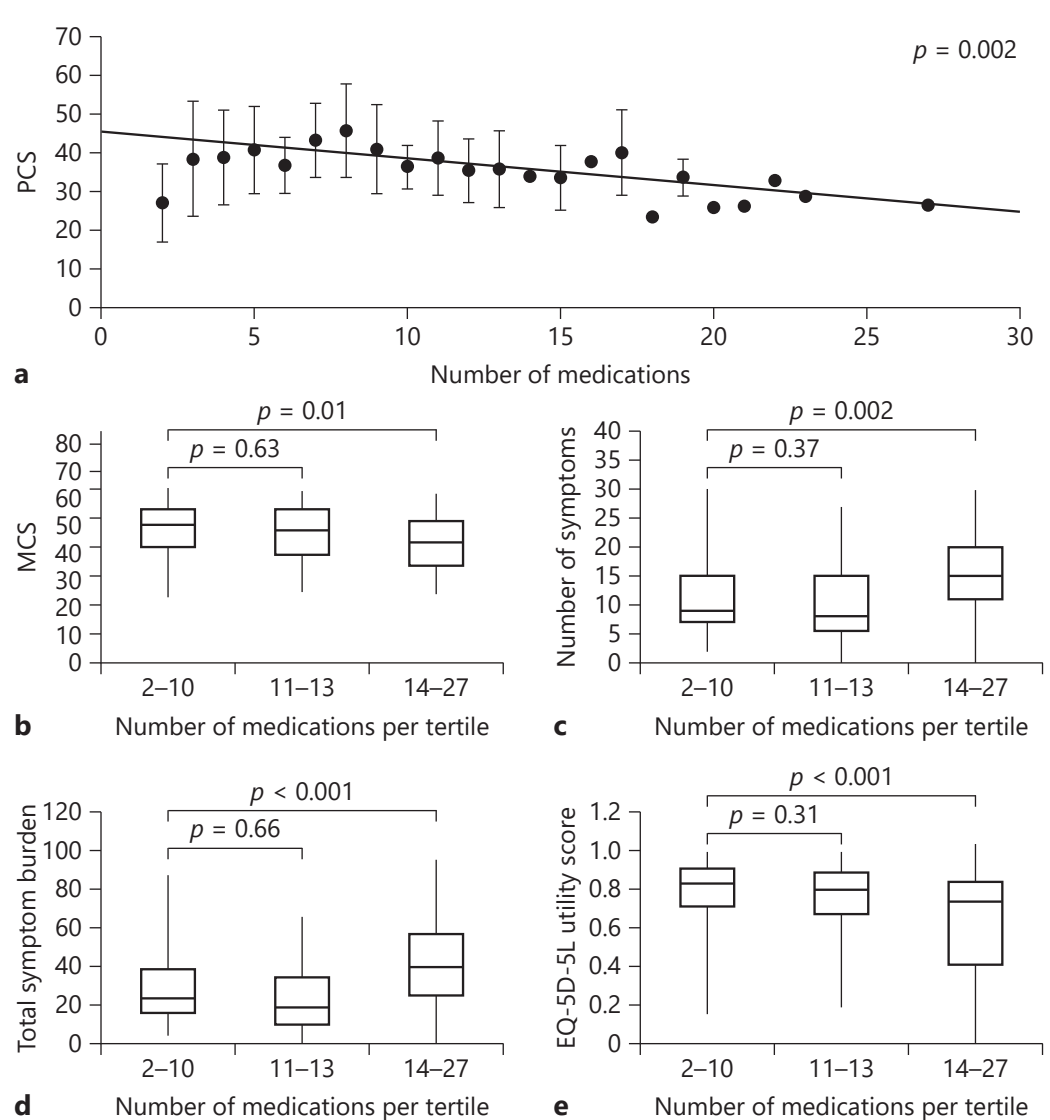

d

Number of medications per tertile

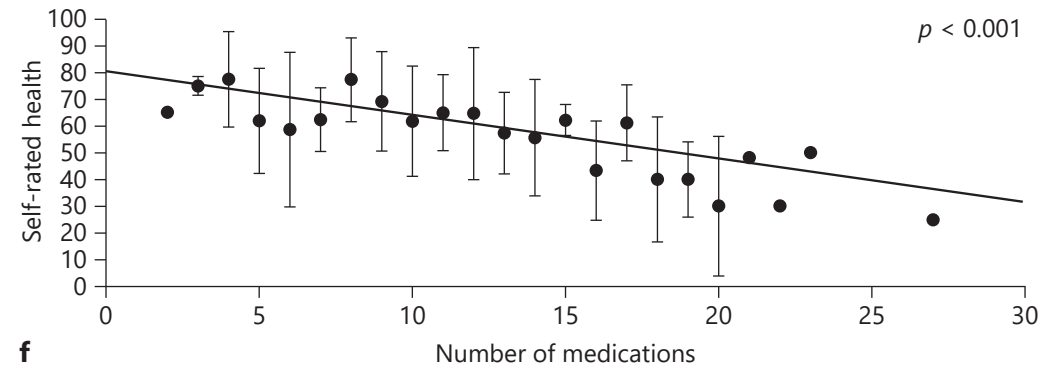

\section{Discussion/Conclusion}

Results for the sensitivity analyses were comparable to the result from the main analyses apart from total symptom burden in the complete case analysis. In this adjusted analysis, a smaller difference in total symptom burden was observed for patients with 14-27 medications (difference 9.1 95\% CI: -0.8-19.1) and a larger difference for patients with 11-13 medications (difference $-7.495 \% \mathrm{CI}:-17.5-2.8)$ than patients with $2-10$ medications, respectively (online suppl. Table 1 ; see www.karger.com/doi/10.1159/000518454 for all online suppl. material)
We are the first study that extensively investigated the association between the number of types of medications and HRQoL in dialysis patients. At 3 months after dialysis initiation, patients were prescribed on average 12 medications and almost all met the criteria for polypharmacy. We found an inverse linear association between the number of medications and PCS score and self-rated health. In addition, patients with 14-27 medications reported worse MCS scores and EQ-5D-5L utility scores and a higher number of symptoms and total symptom burden than pa- 
tients with 2-10 medications. We found no significant differences in MCS score, number of symptoms, total symptom burden, and EQ-5D-5L utility score between patients with 11-13 and 2-10 medications. This suggests that medication regimens are well tolerated up to a certain threshold. If this threshold is exceeded, the number of medications can start to affect HRQoL negatively. Assuming a clinically important difference of 3 points in SF-12 summary scores, a clinically relevant difference is observed in PCS score for patients whose number of medications differs by 5 or more medications and in MCS score between patients with 14-27 and 2-10 medications.

The impact of medication on HRQoL can be conceived of as an equilibrium between the therapeutic benefits and the burden of medication. This burden can be broadly divided into 4 domains based on the domains presented by Mohammed et al. [34]. First, adhering to medication regimens is an arduous task for patients that becomes increasingly demanding with more medication [11]: a higher number of medications require more procedures to administer all medication, augment the number of drug intake moments, complicate keeping track when to take which medication, and may disrupt patients' daily lives, especially when medication must be taken during the day $[34,35]$. Second, medication, and particularly polypharmacy, frequently causes additional symptoms through adverse events. These symptoms can restrict patients in their daily activities, cause mental distress, or lead to emergency room visits or hospitalization [13, 34, 36, 37]. Third, patients can find the number of medications itself and medication characteristics like taste, size, and route of administration burdensome [34, 38]. Finally, patients can consider the interaction with their physicians regarding their medication burdensome. Patients frequently have difficulties comprehending information about their medication and can feel reproached if physicians point out that they are taking their medication incorrectly [34].

An additional problem of polypharmacy is that nonadherence among patients is high, especially for patients who are more negligent in their self-care $[35,39]$. This not only increases the likelihood of complications, which in itself reduce HRQoL, but also amplifies the number of medications required to treat complications, thereby possibly further reducing HRQoL.

Their large number of medications makes dialysis patients particularly prone to the above-mentioned factors, resulting in a sizeable medication burden. However, patients may not be aware of the therapeutic benefits of their medication since much of their medication is primarily prescribed to prevent the development or progression of

Polypharmacy and Quality of Life Dialysis Patients complications of kidney disease rather than alleviate symptoms [11, 40]. Hence, patients may, overall, predominantly be aware of the burden of medication.

A class of medication, which most notably may contribute to this burden, are phosphate binders since they taste unpleasant and are hard to swallow due to their size, and patients usually have to take multiple tablets up to 3 times a day, thereby significantly increasing the number of intake moments and pill burden [41, 42]. Interestingly enough, it appears as if dialysis modality does not affect the medication burden as haemodialysis and peritoneal dialysis patients are equally distributed across the medication tertiles. We did not distinguish between high-flux and low-flux haemodialysis nor online-haemodiafiltration and haemodialysis because they are not associated with benefits on various domains on quality of life compared to conventional haemodialysis $[43,44]$.

Previous studies among dialysis patients also report that polypharmacy is associated with lower quality of life. A US study in 233 haemodialysis patients reports a correlation between a higher number of medications and pill burden and lower PCS but not MCS scores. However, they did not report any effect estimates nor adjusted their results for possible confounders and only included oral and parenteral medications [16]. A Palestinian study in 267 haemodialysis patients reports that using 4 or more chronic medications was associated with worse EQ-5D-5L utility scores and self-rated health [17] and a Brazilian study in 200 haemodialysis patients found that using more medication was associated with worse outcomes for the physical functioning and mental health domains of the SF-36 [18]. Neither the Palestinian nor the Brazilian study describes which medication was included or gives detailed information on the number of medications their participants use: the former dichotomizes medication use in more or less than 4 medications, while the latter does not provide any information on medication use at all. The Brazilian study also did not report SF-36 summary scores. This makes comparing our results with these 2 studies challenging.

Our study has several strengths. First, we measured HRQoL in various ways, using multiple validated questionnaires with different measurement properties. Second, results were adjusted for several potential confounders, including comorbidity. At first sight, comorbidity may have biased our results due to confounding by indication. Since the prescription of medication and comorbidity are strongly correlated, the number of medications could be considered a proxy for the amount of comorbidity. In that case, not more medication but more comorbidity would explain lower HRQoL. Nevertheless, after ad- 
justing for comorbidity with the CCI, observed HRQoL scores remained lower for patients using more medication, suggesting that medication itself, despite the possible role of comorbidity, also affects HRQoL. Third, to the best of our knowledge, this is the first study to examine the association between polypharmacy and HRQoL, which included both patients on haemodialysis and peritoneal dialysis. Fourth, we included both prescribed-as-needed and non-systemic medication in the number of medications. Fifth, multiple imputation reduced selection bias by limiting the number of excluded patients. Last, our results remained robust in sensitivity analyses, indicating that multiple imputation and using 6-month questionnaires did not introduce a significant information bias.

The main limitation of this study is that the cross-sectional design prevents us from demonstrating any causal relationship between polypharmacy and HRQoL. Other limitations are possible selection bias as we only used a subset of DOMESTICO patients or residual bias in observed associations, for example, through comorbidity that is unaccounted for in the CCI. Furthermore, we did not study several medication-related factors that also could affect HRQoL such as drug types, self-medication, and pill burden patients' attitudes toward medication. Especially pill burden may be an important medication-related determinant of HRQoL for dialysis patients as this is usually much higher than the number of medications [16].

In conclusion, this study provides evidence that a higher number of types of medications are associated with worse HRQoL in dialysis patients. This suggests that it is relevant to weigh expected therapeutic benefits of medication against their possible harmful effects on HRQoL. An unfavourable balance between expected benefits and an impact on HRQoL may be ground to be more liberal with adhering to clinical guidelines, especially for patients with a limited life expectancy or for whom a kidney transplant is unattainable. Further research should focus on corroborating our results in a longitudinal design and disentangling how and which medications and medication-related factors affect HRQoL. Most importantly, interventions should be developed for this vulnerable population that aim to reduce the medication burden, without disregarding long-term outcomes.

\section{Acknowledgments}

We would like to thank all patients, investigators, and study nurses from participating centres of the DOMESTICO study for their involvement in DOMESTICO, in particular David Severs (Erasmus Medical Centre, Rotterdam, The Netherlands) and Rob
Klaassen and Marleen van Agtmael (Zaans Medical Centre, Zaandam, The Netherlands). DOMESTICO committee members and investigators: Paul Leurs, Admiraal de Ruyter Hospital Goes; Mario Korte, Albert Schweitzer Hospital Dordrecht; Anita Schrander, Alrijne Hospital; Nynke Cnossen, Amphia Hospital Breda; Frans van Ittersum, Anna Bonenkamp and Brigit van Jaarsveld, Amsterdam University Medical Centers (VU University, Amsterdam) and Diapriva Dialysis Center Amsterdam; An de Vriese, AZ St-Jan Brugge (Belgium); Joy Lips, Bernhoven Uden; Herman Krepel, Bravis Hospital Roosendaal; Marc ten Dam, Canisius-Wilhelmina Hospital Nijmegen; Stijn Konings, Catharina Hospital Eindhoven; Anita van Eck van der Sluijs, Deventer Hospital; Lotte Lips, Dialysis Center Beverwijk; Akin Özyilmaz, Dialysis Centre Groningen; Aegida Neradova, Dianet Amsterdam; Frans Boereboom, Dianet Utrecht; Sadie van Esch, Elisabeth-TweeSteden Hospital Tilburg; Christopher Susanto, Elkerliek Hospital; Fenna van Breda, Elyse Clinics; Ewout Hoorn and David Severs, Erasmus Medical Center Rotterdam; Arnold Boonstra, FlevoHospital Almere; Robert Nette, Franciscus Gasthuis \& Vlietland Rotterdam; Yolanda Vermeeren, Gelre Hospitals Apeldoorn; Daphne Ijpelaar, Groene Hart Hospital Gouda; Nienke Hommes, Haaglanden Medical Center The Hague; Marjolein van Buren, Haga Hospital The Hague; Julia Hofstra, Hospital Gelderse Vallei Ede; Sabine Diepeveen, Isala Zwolle; Ellen Hoogeveen, Jeroen Bosch Hospital's-Hertogenbosch; Tom Cornelis, Jessa Hospital Hasselt (Belgium); Siska Boorsma, Laurentius Hospital Roermond; Joris Rotmans, Leiden University Medical Center; Arjan van Alphen, Maasstad Hospital Rotterdam; Marc Hemmelder Elisabeth Litjens and Bianca Zomer, Maastricht UMC+; Wilbert Janssen, Martini Hospital Groningen; Arno Kuijper and Charles Beerenhout, Máxima Medical Center Veldhoven; Louwine Bierma, Medical Center Leeuwarden; Hans Brink and Renate Wijering, Medical Spectrum Twente Enschede; Renate Bosma, Niercentrum Midden Nederland Amersfoort; Lars Penne, Northwest Clinics Alkmaar; Carola de Fijter and Harald Brulez, OLVG Amsterdam; Henk van Hamersvelt, Radboudumc Nijmegen; Bas Huisman, Reinier de Graaf Gasthuis Delft; Menno Kooistra and Jacobien Verhave, Rijnstate Arnhem; Gijs van Kempen, Saxenburgh Group; Inge Klein, Slingeland Hospital Doetinchem; Caroline Douma, Spaarne Gasthuis Hoofddorp; Willem Jan Bos, St. Antonius Hospital Nieuwegein; Jaap-Jan Snoep, Tergooi Hilversum; Janneke Mulder, Treant Zorggroep Emmen; Casper Franssen, University Medical Center Groningen; Alferso Abrahams, Marianne Verhaar, and Sanne Vonk, University Medical Center Utrecht; Anton Luik, VieCuri Medical Center Venlo; Rob Klaassen and Anne van Tellingen, Zaans Medical Center Zaandam; Margriet Dekker, Ziekenhuisgroep Twente; Anne Weenink, ZorgSaam Hospital Terneuzen; Mariëlle Krekels, Zuyderland Sittard, Wanda Konijn, Dutch Kidney Patients Association (NVN); and Marc ten Dam, Nefrovisie Quality Center for Nephrology.

\section{Statement of Ethics}

This study was approved by the Medical Research Ethics Committee of the Amsterdam UMC, Location VU Medical Centre (NL63277.029.17), and performed in accordance with the Declaration of Helsinki. All patients provided written informed consent before they were enrolled in the study. 


\section{Conflict of Interest Statement}

A.A.B., A.E.S., A.C.A., and B.C.J. are involved in the DOMESTICO study (Netherlands Trial Register identifier: NL6519). All other authors have no conflicts of interest to declare.

\section{Funding Sources}

The Dutch nOcturnal and hoME dialysis Study To Improve Clinical Outcomes is funded by grants of ZonMw (Grant No. 843004116), Fresenius Medical Care Deutschland GmbH, Baxter Netherlands BV, AstraZeneca, and Dirinco. The grant of ZonMw is provided from the "Health care efficiency research" programme. ZonMw has independently peer reviewed the study protocol. The sponsors had no role in the design, the conduct of the study, and the writing of the manuscript.

\section{Author Contributions}

A.A.B., A.E.S. A.C.A, and B.C.J. designed, secured funding, and set up the DOMESTICO study; J.M.T.C., A.A.B., and B.J. designed this study; A.A.B., A.E.S., J.A.B., A.O. A.H.B., A.C.A., and B.C.J. recruited patients; J.M.T.C. collected and analysed the data; J.M.T.C., A.A.B., A.C.A., and B.C.J. interpreted the results; and J.M.T.C. drafted the manuscript and made tables and figures. All authors provided intellectual content and critically edited, revised, and approved the manuscript.

\section{Data Availability Statement}

The data underlying this article are subject to an embargo of 12 months after completion of the DOMESTICO study. Once the embargo expires the data will be available upon reasonable request.

\section{References}

1 van Sandwijk MS, Al Arashi D, van de Hare FM, van der Torren JMR, Kersten MJ, Bijlsma JA, et al. Fatigue, anxiety, depression and quality of life in kidney transplant recipients, haemodialysis patients, patients with a haematological malignancy and healthy controls. Nephrol Dial Transplant. 2019 May 1;34(5): 833-8

2 Jansz TT, Bonenkamp AA, Boereboom FTJ, van Reekum FE, Verhaar MC, van Jaarsveld BC. Health-related quality of life compared between kidney transplantation and nocturnal hemodialysis. PLoS One. 2018;13(9): e0204405.

3 Almutary H, Bonner A, Douglas C. Symptom burden in chronic kidney disease: a review of recent literature. J Ren Care. 2013 Sep 1;39(3): $140-50$.

4 van der Willik EM, Hemmelder MH, Bart HAJ, van Ittersum FJ, Hoogendijk-van den Akker JM, Bos WJW, et al. Routinely measuring symptom burden and health-related quality of life in dialysis patients: first results from the Dutch registry of patient-reported outcome measures. Clin Kidney J. 2020;14(6) 1535-44.

5 Palmer SC, Hanson CS, Craig JC, Strippoli GF, Ruospo M, Campbell K, et al. Dietary and fluid restrictions in CKD: a thematic synthesis of patient views from qualitative studies. Am J Kidney Dis. 2015 Apr 1;65(4):559-73.

6 Masud A, Costanzo EJ, Zuckerman R, Asif A. The complications of vascular access in hemodialysis. Semin Thromb Hemost. 2018 Feb;44(1):57-9.

7 Perl J, Fuller DS, Bieber BA, Boudville N, Kanjanabuch T, Ito Y, et al. Peritoneal dialysis-related infection rates and outcomes: results from the Peritoneal Dialysis Outcomes and Practice Patterns Study (PDOPPS). Am J Kidney Dis. 2020 Jul 7;76(1):42-53.

8 Alvarez L, Brown D, Hu D, Chertow GM, Vassalotti JA, Prichard S. Intradialytic symp- toms and recovery time in patients on thriceweekly in-center hemodialysis: a cross-sectional online survey. Kidney Med. 2019 Dec 20;2(2):125-30

9 Montiel-Luque A, Núñez-Montenegro AJ, Martín-Aurioles E, Canca-Sánchez JC, ToroToro MC, González-Correa JA, et al. Medication-related factors associated with health-related quality of life in patients older than 65 years with polypharmacy. PLoS One. 2017; 12(2):e0171320-e20

10 Woźniak I, Kolonko A, Chudek J, Nowak Ł, Farnik M, Więcek A. Influence of polypharmacy on the quality of life in stable kidney transplant recipients. Transplant Proc. 2018 Jul 01;50(6):1896-9.

11 Krska J, Morecroft CW, Poole H, Rowe PH. Issues potentially affecting quality of life arising from long-term medicines use: a qualitative study. Int J Clin Pharm. 2013 Dec 1;35(6): 1161-9.

12 Laatikainen O, Sneck S, Bloigu R, Lahtinen M, Lauri T, Turpeinen M. Hospitalizations due to adverse drug events in the elderly-a Retrospective Register Study. Front Pharmacol. 2016;7:358-8.

13 Lalic S, Sluggett JK, Ilomäki J, Wimmer BC, Tan EC, Robson L, et al. Polypharmacy and medication regimen complexity as risk factors for hospitalization among residents of long-term care facilities: a prospective cohort study. J Am Med Dir Assoc. 2016 Nov 1; 17(11):1067.e1-e6.

14 Manley HJ, Garvin CG, Drayer DK, Reid GM, Bender WL, Neufeld TK, et al. Medication prescribing patterns in ambulatory haemodialysis patients: comparisons of USRDS to a large not-for-profit dialysis provider. Nephrol Dial Transplant. 2004;19(7):18428.

15 McIntyre C, McQuillan R, Bell C, Battistella M. Targeted deprescribing in an outpatient hemodialysis unit: a Quality Improvement
Study to decrease polypharmacy. Am J Kidney Dis. 2017 Nov 1;70(5):611-8.

16 Chiu YW, Teitelbaum I, Misra M, de Leon EM, Adzize T, Mehrotra R. Pill burden, adherence, hyperphosphatemia, and quality of life in maintenance dialysis patients. Clin J Am Soc Nephrol. 2009 Jun;4(6): 1089-96.

17 Zyoud SH, Daraghmeh DN, Mezyed DO, Khdeir RL, Sawafta MN, Ayaseh NA, et al. Factors affecting quality of life in patients on haemodialysis: a cross-sectional study from Palestine. BMC Nephrol. 2016 Apr 27;17(1): 44.

18 Teles F, Amorim de Albuquerque AL, Freitas Guedes Lins IK, Carvalho Medrado P, Falcão Pedrosa Costa A. Quality of life and depression in haemodialysis patients. Psychol Health Med. 2018 Oct 21;23(9):106978.

19 van Eck van der Sluijs A, Bonenkamp AA Dekker FW, Abrahams AC, van Jaarsveld BC. Dutch nOcturnal and hoME dialysis Study to Improve Clinical Outcomes (DOMESTICO): rationale and design. BMC Nephrol. 2019 Sep $18 ; 20(1): 361$

20 Derington CG, Gums TH, Bress AP, Herrick JS, Greene TH, Moran AE, et al. Association of total medication burden with intensive and standard blood pressure control and clinical outcomes: a secondary analysis of SPRINT. Hypertension. 2019;74(2):26775.

21 Masnoon N, Shakib S, Kalisch-Ellett L, Caughey GE. What is polypharmacy? A systematic review of definitions. BMC Geriatr. 2017 Oct 10;17(1):230.

22 van der Willik EM, Leegte $M$, van Ittersum FJ, Prantl K, Bart HAJ, Dekker FW, et al. FP64 First Dutch registry of patient-reported outcome measures (PROMS) has a low response in dialysis patients. Nephrol Dial Transplant. 2018;33(Suppl 1_1):i262-i62. 
23 van der Willik EM, Meuleman Y, Prantl K, van Rijn G, Bos WJW, van Ittersum FJ, et al. Patient-reported outcome measures: selection of a valid questionnaire for routine symptom assessment in patients with advanced chronic kidney disease: a four-phase mixed methods study. BMC Nephrol. 2019;20(1): $344-4$.

24 Ware J, Kosinski M, Keller SD. A 12-item Short-Form Health Survey: construction of scales and preliminary tests of reliability and validity. Med Care. 1996;34(3):220-33.

25 Ware JE, Sherbourne CD. The MOS 36-item Short-Form Health Survey (SF-36): I. Conceptual framework and item selection. Med Care. 1992;30(6):473-83.

26 Ware J, Kosinski M, Turner-Bowker D, Gandeck MS. User's manual for the SF-12v2 health survey (with a supplement documenting SF-12 health survey). 2007.

27 Hall YN, Larive B, Painter P, Kaysen GA, Lindsay RM, Nissenson AR, et al. Effects of six versus three times per week hemodialysis on physical performance, health, and functioning: frequent hemodialysis network (FHN) randomized trials. Clin J Am Soc Nephrol. 2012;7(5):782.

28 Weisbord SD, Fried LF, Arnold RM, Rotondi AJ, Fine MJ, Levenson DJ, et al. Development of a symptom assessment instrument for chronic hemodialysis patients: the dialysis symptom index. J Pain Symptom Manage. 2004 Mar 1;27(3):226-40.

29 Herdman M, Gudex C, Lloyd A, Janssen M, Kind P, Parkin D, et al. Development and preliminary testing of the new five-level version of EQ-5D (EQ-5D-5L). Qual Life Res. 2011 Dec 1;20(10):1727-36.
30 Versteegh MM, Vermeulen KM, Evers SMAA, de Wit GA, Prenger R, Stolk EA. Dutch tariff for the five-level version of EQ5D. Value Health. 2016 Jun;19(4):343-52.

31 Kramer A, Boenink R, Noordzij M, Bosdriesz JR, Stel VS, Beltrán P, et al. The ERA-EDTA registry annual report 2017: a summary. Clin Kidney J. 2020;13(4):693-709.

32 Fried L, Bernardini J, Piraino B. Comparison of the Charlson comorbidity index and the Davies score as a predictor of outcomes in PD patients. Perit Dial Int. 2003 Nov 1;23:56873.

33 Eekhout I, de Vet HC, Twisk JW, Brand JP, de Boer MR, Heymans MW. Missing data in a multi-item instrument were best handled by multiple imputation at the item score level. J Clin Epidemiol. 2014 Mar;67(3):335-42.

34 Mohammed MA, Moles RJ, Chen TF. Medication-related burden and patients' lived experience with medicine: a systematic review and metasynthesis of qualitative studies. BMJ Open. 2016;6(2): e010035.

35 Neri L, Martini A, Andreucci VE, Gallieni M, Rey LA, Brancaccio D. Regimen complexity and prescription adherence in dialysis patients. Am J Nephrol. 2011;34(1):71-6.

36 Chan L, Saha A, Poojary P, Chauhan K, Naik $\mathrm{N}$, Coca $\mathrm{S}$, et al. National trends in emergency room visits of dialysis patients for adverse drug reactions. Am J Nephrol. 2018;47(6): 441-9.

37 Schenker Y, Park SY, Jeong K, Pruskowski J, Kavalieratos D, Resick J, et al. Associations between polypharmacy, symptom burden, and quality of life in patients with advanced, life-limiting illness. J Gen Intern Med. 2019 Apr 1;34(4):559-66.
38 Tesfaye WH, McKercher C, Peterson GM, Castelino RL, Jose M, Zaidi STR, et al. Medication adherence, burden and health-related quality of Llife in adults with predialysis chronic kidney disease: a Prospective Cohort Study. Int J Environ Res Public Health. 2020; 17(1):371.

39 Ghimire S, Castelino RL, Lioufas NM, Peterson GM, Zaidi ST. Nonadherence to medication therapy in haemodialysis patients: a systematic review. PLoS One. 2015;10(12): e0144119.

40 Marin JG, Beresford L, Lo C, Pai A, EspinoHernandez G, Beaulieu M. Prescription patterns in dialysis patients: differences between hemodialysis and peritoneal dialysis patients and opportunities for deprescription. Can J Kidney Health Dis. 2020 Jan 1;7: 2054358120912652.

41 Karamanidou C, Clatworthy J, Weinman J, Horne R. A systematic review of the prevalence and determinants of nonadherence to phosphate binding medication in patients with end-stage renal disease. BMC Nephrol. 2008 Jan 31;9:2.

42 Iwashita $Y$, Ohya M, Kunimoto S, Iwashita $Y$, Mima T, Negi S, et al. A survey of drug burden in patients undergoing maintenance hemodialysis in Japan. Intern Med. 2018;57(20): 2937-44.

43 Unruh M, Benz R, Greene T, Yan G, Beddhu $S$, DeVita M, et al. Effects of hemodialysis dose and membrane flux on health-related quality of life in the HEMO Study. Kidney Int. 2004 Jul;66(1):355-66.

44 Mazairac AH, de Wit GA, Grooteman MP, Penne EL, van der Weerd NC, den Hoedt CH, et al. Effect of hemodiafiltration on quality of life over time. Clin J Am Soc Nephrol. 2013; 8(1):82-9. 